providers saw definite advantages of diagnostic testing in terms of patient management, they also expressed reservations about the additional work load this would necessitate.

Conclusions There are high levels of receptivity for diagnostic testing among providers and communities with certain caveats regarding specimens and timing.

\section{S16.3 CLINICAL MANAGEMENT OF STI IN PREGNANCY}

John Kinuthia*. Kenyatta National Hospital and University of Nairobi, Nairobi, Kenya

\subsection{6/sextrans-2015-052270.73}

Sexually transmitted infections (STIs) in pregnancy prevalent in many resource limited settings are associated with adverse pregnancy outcomes that include spontaneous abortion, stillbirths, preterm rupture of membranes, premature delivery and postpartum endometritis. Antenatal clinic attendance provides opportunity to screen the women for STIs, offer prompt treatment for curable STIs or prophylaxis for viral infections such as HIV and to promote prevention. Although routine screening for syphilis and HIV is recommended as part of antenatal care (ANC), optimal screening rates have not been achieved. This is due antenatal clinic non-attendance, non-availability of screening tests and where available, health system inefficiencies such long wait time before test results are available or need for women to make return visits to receive results. Use of point-of-care tests that detect syphilis and HIV have been shown to increase the number of women tested for HIV and syphilis, are inexpensive, easy to use and do not require sophisticated laboratory. In resource limited settings, prompt detection and treatment of the other STIs is hampered by lack of screening tests. Often, health providers rely on syndromic approach to diagnose and manage STIs. This approach alleviates need for laboratory services but misses women with asymptomatic infections and has poor diagnostic performance. Pregnant women with STIs are at an increased risk of acquiring HIV and transmitting the infection to their infants. Increased availability of HIV testing and provision of effective PMTCT regimens has dramatically reduced the number of new paediatric HIV infections. However, STIs, apart from HIV, have not been viewed as a public health priority and adequate resources allocated to combat them. To optimise maternal and infant outcomes, there is need to prioritise development of low cost tests to detect STIs during pregnancy and to ensure women are provided with effective treatment promptly.

\section{S16.4 POINT-OF-CARE TESTING AND TREATMENT OF SEXUALLY TRANSMITTED INFECTIONS TO IMPROVE PREGNANCY OUTCOMES IN HIGH-BURDEN SETTINGS}

Andrew Vallely*. UNSW Australia, Sydney, Australia

\subsection{6/sextrans-2015-052270.74}

Chlamydia trachomatis, Neisseria gonorrhoeae, Trichomonas vaginalis and bacterial vaginosis are highly prevalent among pregnant women in many low- and middle-income countries, and have been associated with increased risk of adverse maternal and neonatal health outcomes, particularly preterm birth and low birth weight. In a majority of women, these curable genital infections are asymptomatic and therefore remain undiagnosed and untreated in pregnancy because of a lack of suitable diagnostic technologies. Newly available, easy to use and highly accurate point-of-care assays for chlamydia, gonorrhoea, trichomonas and bacterial vaginosis are now available for use in routine clinical settings. A large-scale field trial to evaluate antenatal point-ofcare testing and immediate treatment of curable sexually transmitted and genital infections to improve pregnancy outcomes in high-burden low-income settings will start recruitment in Papua New Guinea in early 2016.

\section{S17 - HPV and HIV: new evidence on the infection risks and strategies to prevent HPV-related disease among HIV-infected people}

\section{S17.1 CERVICAL CANCER PREVENTION IN HIV-INFECTED WOMEN}

Jennifer Smith*. University of North Carolina, Chapel Hill, USA

\subsection{6/sextrans-2015-052270.75}

HIV-infected women are living longer and are at a higher risk of acquiring cervical cancer. More data comparing cervical cancer screening and treatment strategies in these women are urgently needed. WHO guidelines on optimal methods to screen and treat cervical cancer are available, but there are limited data to inform their potential modification for HIV-infected women. Even fewer data are available which compare the efficacy of treatment methods (loop electrosurgical excision procedure (LEEP) and cryotherapy) and differences in cervical disease recurrence between HIV-infected and HIV-uninfected women.

HPV testing appears to be the most sensitive, although less specific for CIN-2+ detection than either VIA and cytology screening. Several studies of HIV-infected women have shown equivalent sensitivity of VIA and of cytology screening for CIN$2+$, although there is variation in results. Consistently across studies, the specificity of cytology appears higher than either VIA or for high-risk HPV testing. Two studies in South Africa and Kenya have suggested that the specificity of HPV testing and VIA for detection of high grade lesions is lower in HIVinfected women with lower CD4 counts; more research is needed to examine if screening test performance varies for HIVinfected women with higher versus lower CD4 counts.

More research in HIV-infected women is needed to examine if LEEP and cryotherapy treatment methods are associated with differences in HIV-viral shedding and in HIV transmission during or shortly after treatment.

HIV-infected adolescents have found to induce high-levels of neutralizing antibody titers following prophylactic HPV vaccination in bridging studies. Studies are not yet available on HPV vaccine efficacy in HIV infected women against either HPVinfection or clinical disease endpoints. 